Western University

Scholarship@Western

Biochemistry Publications

Biochemistry Department

6-12-2012

(1) $\mathrm{H},(15) \mathrm{N}$ and (13)C backbone resonance assignments of the Kelch domain of mouse Keap 1.

Elio Cino

Jingsong Fan

Daiwen Yang

Wing-Yiu Choy

Follow this and additional works at: https://ir.lib.uwo.ca/biochempub

Part of the Biochemistry Commons

Citation of this paper:

Cino, Elio; Fan, Jingsong; Yang, Daiwen; and Choy, Wing-Yiu, "(1)H, (15)N and (13)C backbone resonance assignments of the Kelch domain of mouse Keap1." (2012). Biochemistry Publications. 154.

https://ir.lib.uwo.ca/biochempub/154 


\section{${ }^{1} \mathrm{H},{ }^{15} \mathrm{~N}$ and ${ }^{13} \mathrm{C}$ backbone resonance assignments of the Kelch domain of mouse Keap1}

Elio Cino ${ }^{1}$, Jingsong Fan ${ }^{2}$, Daiwen Yang ${ }^{2}$, and Wing-Yiu Choy ${ }^{1 *}$

${ }^{I}$ Department of Biochemistry, The University of Western Ontario, London, Ontario, Canada N6A 5C1

${ }^{2}$ Department of Biological Sciences, National University of Singapore, 14 Science Drive 4, Singapore 117543

*Corresponding author

E-mail: jchoy4@uwo.ca

Tel: 519-661-3161

Fax: 519-661-3175 


\begin{abstract}
Keap1 is a multi-domain protein that functions as an inhibitor of the transcription factor Nrf2 in the cellular response to oxidative stress. Under normal conditions, Keap1 binds to Nrf2 via its C-terminal Kelch domain and the interaction ultimately leads to the ubiquitin-dependent degradation of Nrf2. It has been proposed that designing molecules to selectively disrupt the Keap1-Nrf2 interaction can be a potential therapeutic approach for enhancing the expression of cytoprotective genes. Here, we reported the ${ }^{1} \mathrm{H},{ }^{13} \mathrm{C}$, and ${ }^{15} \mathrm{~N}$ backbone chemical shift assignments of the Kelch domain of mouse Keap1. Further, unlabeled Nrf2 peptide containing the Kelch-binding motif was added to the ${ }^{15} \mathrm{~N}$-labeled Kelch sample. ${ }^{1} \mathrm{H}-{ }^{15} \mathrm{~N}$ HSQC spectra of the protein in the absence and presence of an equimolar concentration of the Nrf2 peptide were presented. A significant number of resonance signals were shifted upon addition of the peptide, confirming the protein-peptide interaction. The results here will not just facilitate the further studies of the binding between Keap1 and Nrf2, it will also be valuable for probing interactions between the Kelch domain and small molecules, as well as a growing list of protein targets that have been identified recently.
\end{abstract}

\title{
Keywords
}

Oxidative stress response; Keap1; Kelch domain; Nrf2; protein-protein interaction 


\section{Biological context}

Keap1 (Kelch-like ECH-associated Protein 1) is a 70-kDa protein that is rich in cysteine. It functions as the repressor of Nrf2 (nuclear factor E2-related factor 2), the key transcription factor that coordinates the cellular responses to oxidative stress. Keap1 is composed of three domains (Itoh et al. 1999). The N-terminal BTB (Broad-Complex, Tramtrack and Bric-a-Brac) domain mediates the protein dimerization and is also responsible for the binding to the Cul3-dependent E3 ubiquitin ligase complex (Itoh et al. 1999). The BTB domain is linked to the C-terminal Kelch domain by the intervening region (IVR), where cysteines that are pivotal for the Keapl functions as chemical sensor are located (Itoh et al. 1999). The C-terminal Kelch domain is responsible for target recognition. It directly interacts with the ETGE and DLG motifs present in the N-terminal Neh2 domain of Nrf2 (Baird and Dinkova-Kostova 2011; Tong et al. 2006; Zipper and Mulcahy 2002). Under unstressed conditions, Keap1 binds to Nrf2 and targets this transcription factor for degradation (Itoh et al. 2004).

The 32-kDa Kelch domain adopts a six-bladed $\beta$-propeller conformation and the structures in complex with peptides derived from Nrf2 that contain the binding motifs have been determined by X-ray crystallography (Lo et al. 2006; Padmanabhan et al. 2006; Tong et al. 2007). Due to the critical roles Keap1 and Nrf2 play in the oxidative stress response mechanism, disrupting their interaction has been proposed as a potential strategy to enhance the expression of cytoprotective genes. The backbone resonance assignments obtained here will facilitate the screening of inhibitors that can disrupt the Keap1-Nrf2 interaction. In addition to Nrf2, several novel targets of the Kelch domain of Keap1 have been identified recently. These include FAC1 (Strachan et al. 2004), PGAM5 (Lo and Hannink 2006), WTX (Major et al. 2007; Camp et al. 2012), prothymosin $\alpha$ (Padmanabhan et al. 2008), p62 (Komatsu et al. 2010) and PALB2 (Ma et al. 2012). The assignments reported here will also serve as important starting points for studying the interaction of Keap1 with these proteins.

\section{Methods and experiments}

\section{Sample preparation}

The mouse Keap1 cDNA (GenBank\# BC055732) was purchased from the American Type Culture Collection (ATCC) and the Kelch domain (residues 324-612) was cloned into the expression vector pDEST17 (Invitrogen). The TEV cleavage recognition sequence, 'ENLYFQG', was introduced between the histidine tag and insert. This vector was transformed into E. coli BL21(DE3). ${ }^{15} \mathrm{~N},{ }^{13} \mathrm{C}$ and ${ }^{2} \mathrm{H}$ labeled protein was produced by growing $E$. coli in deuterated M9 media. Cells adapted in $70 \% \mathrm{D}_{2} \mathrm{O}$ were used to inoculate $1 \mathrm{~L}$ of $\mathrm{M} 9$ prepared in $100 \% \mathrm{D}_{2} \mathrm{O}$. The cells were then grown at $37^{\circ} \mathrm{C}$ and overexpression was induced at an $\mathrm{OD}_{600}$ of 0.6 with $0.5 \mathrm{mM}$ IPTG. After a 60-hour induction, the cells were centrifuged and the pellets were frozen. The N-terminally His-tagged protein was purified by affinity chromatography using Ni Sepharose ${ }^{\mathrm{TM}} 6$ Fast Flow beads (Amersham Biosciences). The tag was cleaved by incubation with His-tagged tobacco etch virus (TEV) protease overnight at $25^{\circ} \mathrm{C}$ and the protein product was purified by passing the mixture through Ni Sepharose ${ }^{\mathrm{TM}} 6$ Fast Flow beads (Amersham Biosciences). For NMR experiments, the purified protein was dialyzed against $50 \mathrm{mM}$ sodium phosphate at $\mathrm{pH} 7$ 
containing $50 \mathrm{mM} \mathrm{NaCl}$ and $1 \mathrm{mM}$ DTT. The samples were then concentrated to $\sim 0.3 \mathrm{mM}$. All samples contained $10 \% \mathrm{D}_{2} \mathrm{O}$ and $1 \mathrm{mM}$ 2,2-dimethyl-2-sila-pentane-5-sulfonic acid (DSS) as ${ }^{1} \mathrm{H}$ and ${ }^{13} \mathrm{C}$ chemical shift references.

\section{Sequential assignment experiments}

NMR experiments for the backbone resonance assignment were conducted at $25^{\circ} \mathrm{C}$ on Varian INOVA $800 \mathrm{MHz}$ (NANUC) and Bruker Avance $800 \mathrm{MHz}$ (Singapore) spectrometers equipped with cryogenic probes. Sequential assignments were obtained from ${ }^{1} \mathrm{H}_{-}{ }^{15} \mathrm{~N}$ TROSY-HSQC, HNCA, HN(CO)CA, HNCACB, HN(CO)CACB and ${ }^{15} \mathrm{~N}-\mathrm{NOESY}-$ HSQC spectra. The data was processed with NMRPipe (Delaglio et al. 1995) and analyzed using CARA (Keller 2004).

\section{Ligand binding experiment}

To demonstrate the binding of the Kelch domain to a mouse Nrf2 peptide (Ac-

${ }^{72}$ AQFQLDEETGEFLP ${ }^{85}-\mathrm{NH}_{2}$; ordered from NEOpeptide), ${ }^{1} \mathrm{H}-{ }^{15} \mathrm{~N}$ HSQC spectra of ${ }^{15} \mathrm{~N}$ labeled Kelch domain $(\sim 200 \mu \mathrm{M})$ were collected on a Varian Inova $600 \mathrm{MHz}$ spectrometer with cryogenic probe (UWO Biomolecular NMR Facility) at $25^{\circ} \mathrm{C}$ in the absence and presence of equimolar concentration of the Nrf2 peptide.

\section{Assignments and data deposition}

The ${ }^{1} \mathrm{H}^{-15} \mathrm{~N}$ TROSY-HSQC of the Kelch domain of Keap1 (Figure 1a) had well dispersed peaks for a protein of this size. We were able to assign $91.3 \%$ of the ${ }^{1} \mathrm{H}^{\mathrm{N}}$ and ${ }^{15} \mathrm{~N}$ resonances of non-proline residues, $90.7 \%$ of all ${ }^{13} \mathrm{C} \alpha$ and $90 \%$ all ${ }^{13} \mathrm{C} \beta$ resonances.

Repetitive sequences of the $\beta$-propeller structure (Figure 1b) made it difficult to obtain a higher percentage of resonance assignments. The ${ }^{1} \mathrm{H},{ }^{15} \mathrm{~N}$ and ${ }^{13} \mathrm{C} \alpha / \beta$ chemical shifts of the backbone resonances have been deposited in the BioMagResBank

(http://www.bmrb.wisc.edu), under BMRB accession number 18353.

Figure 2 shows the residue-specific secondary structure propensity (SSP) scores determined using the program SSP on the basis of the assigned ${ }^{13} \mathrm{C} \alpha / \beta$ chemical shifts (Marsh et al. 2006). The result strongly indicates that the protein has an all- $\beta$ fold (Figure 2 ). This is in good agreement with the crystal structure of the Kelch domain (PDB id: $1 \mathrm{X} 2 \mathrm{~J})$, which shows that the protein adopts a six-bladed $\beta$-propeller conformation (Padmanabhan et al. 2006). The SSP scores we obtained here are also consistent with the DSSP analysis (Kabsch and Sander 1983) and the secondary structure plot (Laskowski 2009) of the crystal structure (Padmanabhan et al. 2006).

The overlay of the ${ }^{1} \mathrm{H}-{ }^{15} \mathrm{~N}$ HSQC spectra of the Kelch domain in the absence and presence of an equimolar concentration of the 14-mer Nrf2 peptide is shown in Figure 3a. A significant number of resonance signals are shifted upon addition of the peptide (Figure 3a). To quantify the magnitude of peak shifts, combined chemical shift changes $(\Delta \omega=$ $\left.\left|\Delta^{15} \mathrm{~N}\right|+\left|\Delta^{1} \mathrm{H}^{\mathrm{N}}\right|\right)$ were calculated, where $\left|\Delta^{15} \mathrm{~N}\right|+\left|\Delta^{1} \mathrm{H}^{\mathrm{N}}\right|$ are the absolute values of resonance frequency change (in $\mathrm{Hz}$ ) in the ${ }^{15} \mathrm{~N}$ and ${ }^{1} \mathrm{H}$ dimensions, respectively. Figure $3 \mathrm{~b}$ shows that many of the traceable assigned residues with $\Delta \omega>50 \mathrm{~Hz}$ (in descending order: G378, N504, G364, G477, G600, G570, G571, G417, G524, G379 and S431) are residues that 
comprise, or are in close proximity, to the binding interface as identified in the crystal structure (Padmanabhan et al. 2006).

\section{Acknowledgments}

This work was supported by an Operating Grant (MOP no. 74679) from the Canadian Institutes of Health Research (CIHR). We are grateful for the use of the NMR facility at National University of Singapore. We would also like to thank the Canadian National High Field NMR Centre (NANUC) for their assistance and use of the facilities. Operation of NANUC is funded by the Natural Science and Engineering Research Council of Canada, and the University of Alberta. 


\section{Figure Legends}

Fig. 1 a ${ }^{1} \mathrm{H}-{ }^{15} \mathrm{~N}$ TROSY-HSQC spectrum and backbone resonance assignment of the ${ }^{2} \mathrm{H} /{ }^{13} \mathrm{C} /{ }^{15} \mathrm{~N}$ labeled Kelch domain of mouse Keap1. The figure was generated using Sparky (Goddard and Kneller). b Protein sequence of the Kelch domain with unassigned resonances colored red and regions with high sequence identities boxed. The starting ' $G$ ' was a non-native residue from the TEV recognition sequence that remained after cleavage.

Fig. 2 Secondary structure propensity (SSP) scores and DSSP analysis of the mouse Kelch domain of Keap1. SSP scores were calculated based upon the ${ }^{13} \mathrm{C} \alpha / \beta$ chemical shifts (Marsh et al. 2006). The crystal structure of the mouse Kelch domain (PDB id: 1X2J) (Padmanabhan et al. 2006) was used for the DSSP analysis (Kabsch and Sander 1983) and generation of the secondary structure cartoon diagram (Laskowski 2009).

Fig. 3 a Overlay of ${ }^{1} \mathrm{H}^{15} \mathrm{~N}$ HSQC spectra in the absence (black) and presence (pink) of an equimolar concentration of the Nrf2 peptide (Ac- $\left.{ }^{72} \mathrm{AQFQLDEETGEFLP}{ }^{85}-\mathrm{NH}_{2}\right)$. b Crystal structure of the mouse Kelch domain of Keap1 (grey) in complex with an Nrf2 peptide (red) (PDB id: 1X2R) (Padmanabhan et al. 2006). Residues with traceable assigned resonances are colored based on their combined absolute proton and nitrogen resonance frequency changes $(\mathrm{Hz})$ upon ligand binding (yellow $<25 \mathrm{~Hz}$, orange $25-50 \mathrm{~Hz}$ and pink $>50 \mathrm{~Hz})$. 


\section{References}

Baird L, and Dinkova-Kostova AT (2011) The cytoprotective role of the Keap1-Nrf2 pathway. Arch Toxicol 85:241-272. doi10.1007/s00204-011-0674-5

Camp ND, James RG, Dawson DW, Yan F, Davison JM, Houck SA, Tang X, Zheng N, Major MB, and Moon RT (2012) Wilms Tumor Gene on X Chromosome (WTX) Inhibits Degradation of NRF2 Protein through Competitive Binding to KEAP1 Protein. J Biol Chem 287:6539-6550. doi10.1074/jbc.M111.316471

Delaglio F, Grzesiek S, Vuister GW, Zhu G, Pfeifer J, and Bax A (1995) NMRPipe: a multidimensional spectral processing system based on UNIX pipes. J Biomol NMR 6:277293.

Goddard TD, Kneller DG, SPARKY 3, University of California, San Francisco

Itoh K, Tong KI, and Yamamoto M (2004) Molecular mechanism activating Nrf2-Keap1 pathway in regulation of adaptive response to electrophiles. Free Radic Biol Med 36:12081213. doi10.1016/j.freeradbiomed.2004.02.075

Itoh K, Wakabayashi N, Katoh Y, Ishii T, Igarashi K, Engel JD, and Yamamoto M (1999) Keap1 represses nuclear activation of antioxidant responsive elements by Nrf2 through binding to the amino-terminal Neh2 domain. Genes Dev 13:76-86. doi10.1101/gad.13.1.76

Kabsch W, and Sander C (1983) Dictionary of protein secondary structure: pattern recognition of hydrogen-bonded and geometrical features. Biopolymers 22:2577-2637. doi10.1002/bip.360221211

Keller R. The Computer Aided Resonance Assignment Tutorial. CANTINA Verlag; 2004

Komatsu M, Kurokawa H, Waguri S, Taguchi K, Kobayashi A, Ichimura Y, Sou YS, Ueno I, Sakamoto A, et al. (2010) The selective autophagy substrate p62 activates the stress responsive transcription factor Nrf2 through inactivation of Keap1. Nat Cell Biol 12:213223. doi10.1038/ncb2021

Laskowski RA (2009) PDBsum new things. Nucleic Acids Res 37:D355-D359. doil0.1093/nar/gkn860

Lo SC, and Hannink M (2006) PGAM5, a Bcl-XL-interacting protein, is a novel substrate for the redox-regulated Keap1-dependent ubiquitin ligase complex. J Biol Chem 281:37893-37903. doi10.1074/jbc.M606539200

Lo SC, Li X, Henzl MT, Beamer LJ, and Hannink M (2006) Structure of the Keap1:Nrf2 interface provides mechanistic insight into Nrf2 signaling. EMBO J 25:3605-3617. doi10.1038/sj.emboj.7601243 
Ma J, Cai H, Wu T, Sobhian B, Huo Y, Alcivar A, Mehta M, Cheung KL, Ganesan S, et al. (2012) PALB2 interacts with KEAP1 to promote NRF2 nuclear accumulation and function. Mol Cell Biol doi10.1128/MCB.06271-11

Major MB, Camp ND, Berndt JD, Yi X, Goldenberg SJ, Hubbert C, Biechele TL, Gingras AC, Zheng N, et al. (2007) Wilms tumor suppressor WTX negatively regulates WNT/betacatenin signaling. Science 316:1043-1046. doi10.1126/science/1141515

Marsh JA, Singh VK, Jia Z, and Forman-Kay JD (2006) Sensitivity of secondary structure propensities to sequence differences between alpha- and gamma-synuclein: implications for fibrillation. Protein Sci 15:2795-2804. doi10.1110/ps.062465306

Padmanabhan B, Nakamura Y, and Yokoyama S (2008) Structural analysis of the complex of Keap1 with a prothymosin alpha peptide. Acta Crystallogr Sect F Struct Biol Cryst Commun 64:233-238. doi10.1107/S1744309108004995

Padmanabhan B, Tong KI, Ohta T, Nakamura Y, Scharlock M, Ohtsuji M, Kang MI, Kobayashi A, Yokoyama S, and Yamamoto M (2006) Structural basis for defects of Keap1 activity provoked by its point mutations in lung cancer. Mol Cell 21:689-700. doi10.1016/j.molcel.2006.01.013

Strachan GD, Morgan KL, Otis LL, Caltagarone J, Gittis A, Bowser R, and Jordan-Sciutto KL (2004) Fetal Alz-50 clone 1 interacts with the human orthologue of the Kelch-like Echassociated protein. Biochemistry 43:12113-12122. doi10.1021/bi0494166

Tong KI, Katoh Y, Kusunoki H, Itoh K, Tanaka T, and Yamamoto M (2006) Keap1 recruits Neh2 through binding to ETGE and DLG motifs: characterization of the two-site molecular recognition model. Mol Cell Biol 26:2887-2900. doi10.1128/MCB.26.8.28872900.2006

Tong KI, Padmanabhan B, Kobayashi A, Shang C, Hirotsu Y, Yokoyama S, and Yamamoto M (2007) Different electrostatic potentials define ETGE and DLG motifs as hinge and latch in oxidative stress response. Mol Cell Biol 27:7511-7521. doi10.1128/MCB.00753-07

Zipper LM, and Mulcahy RT (2002) The Keap1 BTB/POZ dimerization function is required to sequester Nrf2 in cytoplasm. J Biol Chem 277:36544-36552.

doi10.1074/jbc.M206530200 


\section{$\mathbf{a}$}

128.0

132.0

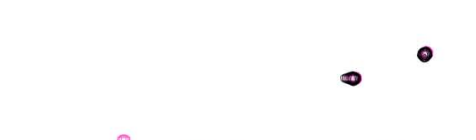

$-0.80$

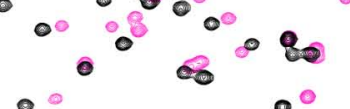<smiles>O=S(=O)(O)O[Na]</smiles>

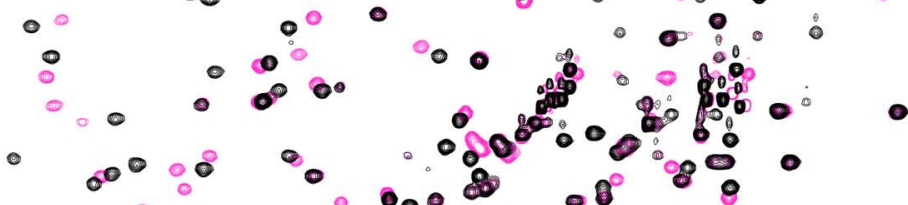
- $0.800^{\circ} \circ 0^{\circ}$

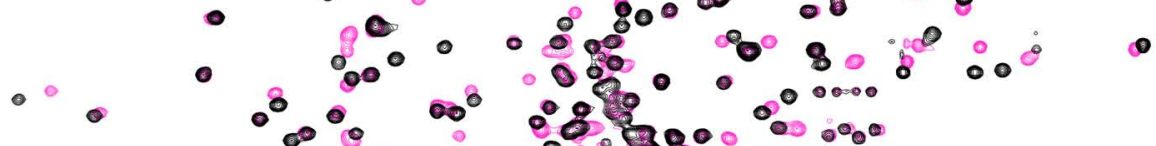

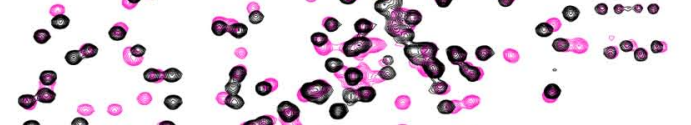

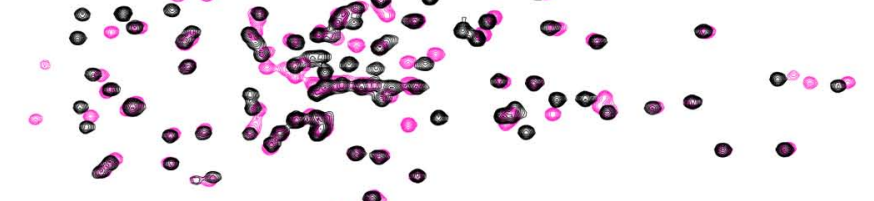

$$
\begin{aligned}
& \text { - 8. } 0.8 \\
& -0^{\circ} 0
\end{aligned}
$$

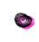

7.0

6.0

b

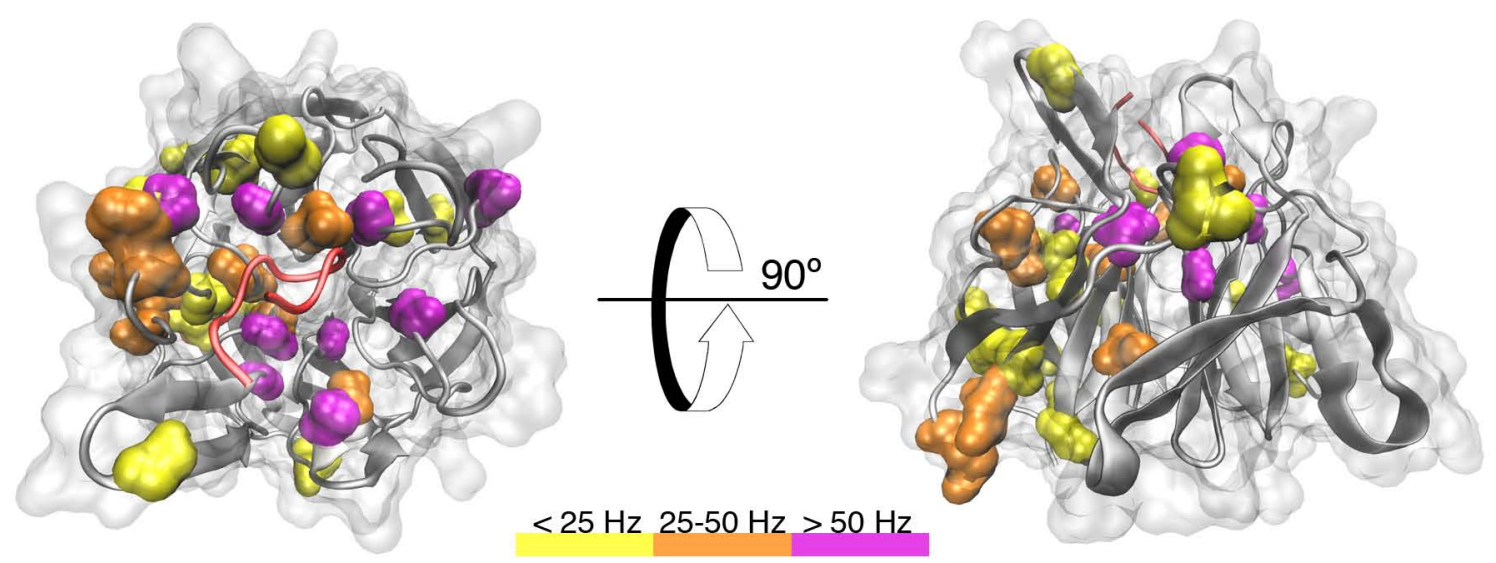




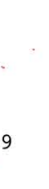

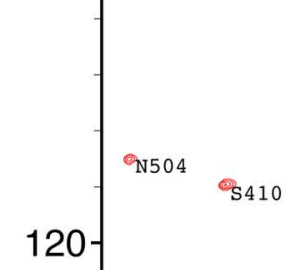

b
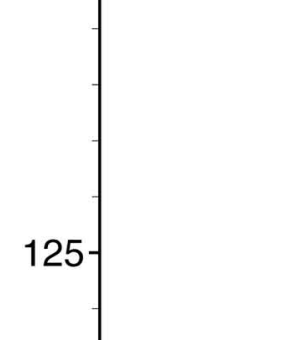

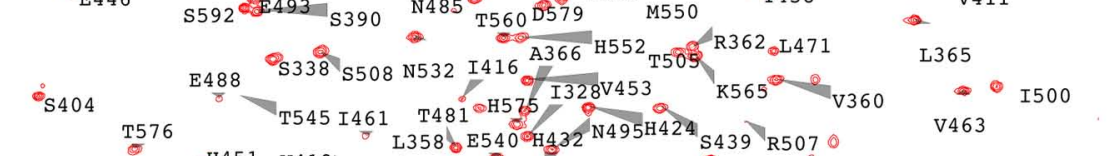

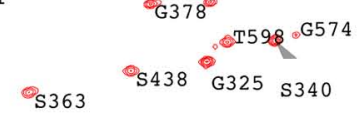

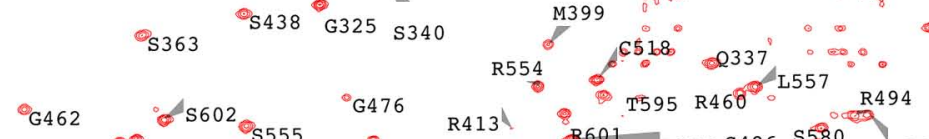
Q402 $\quad$ E449

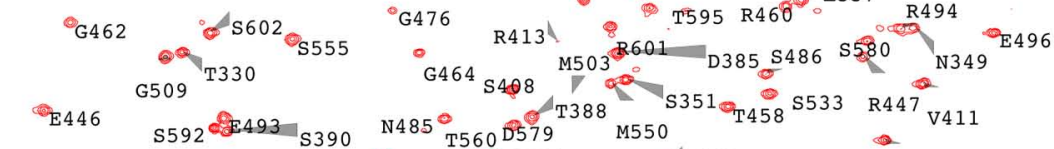

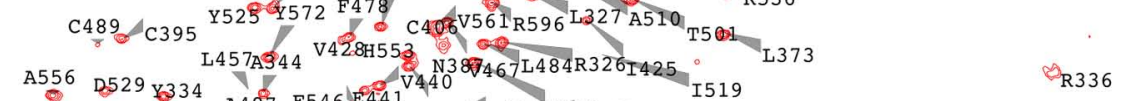

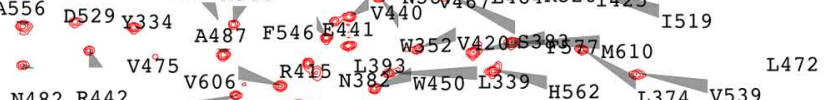

N482 R442 A376 E34 F53 ONA3 H437\% 483 L

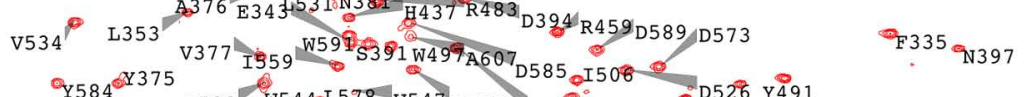

Y584

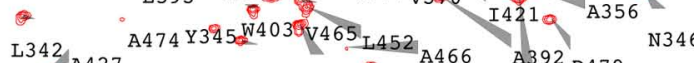

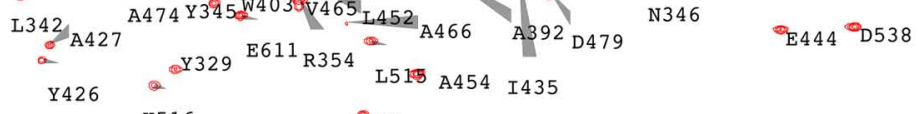

Q5469 ${ }_{\mathrm{D} 422}^{\mathrm{D} 3899_{\mathrm{V} 512}^{\mathrm{L}+468}} \mathrm{H}_{436}$

130

A407 9521

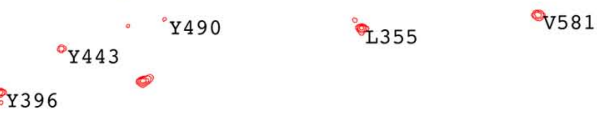

P331

10

9

8

7

$\delta\left({ }^{1} \mathrm{H}\right)[\mathrm{ppm}]$

324

340

360

380

GVGRL I YTAGGYFRQSLSYLEAYNPSNGSWLRLADLQVPRSGLAGCVVGGLLYAVGGR $400 \quad 420$

NNSPDGNTDSSALDCYNPMTNQWSPCASMSVPRNR I GVGVIDGH IYAVGGSHGC I HHS 440 460 480

SVERYEPERDEWHLVAPMLTRRIGVGVAVLNRLLYYAVGGFDGTNRLNSAECYYPERNE 500 520 540

WRM I TPMNT I RSGAGVCVL HNCIYAAGGYDGQDQLNSVERYDVETETWTFVAPMRHHR 